\title{
Visualization and manipulation of actin cytoskeleton with small-molecular probes
}

Takeru Takagi ${ }^{1}$, Tasuku Ueno*1, Keisuke Ikawa4, Daisuke Asanuma ${ }^{2}$, , Yusuke Nomura$^{1}$, Shin-nosuke Uno ${ }^{2}$, Toru Komatsu ${ }^{1}$, Mako Kamiya ${ }^{2}$, Kenjiro Hanaoka ${ }^{1}$, Chika Okimura6, Yoshiaki Iwadate, ${ }^{6}$ Kenzo Hirose ${ }^{2}, 7$, Tetsuo Nagano ${ }^{3}$, Kaoru Sugimura ${ }^{4}$, and Yasuteru Urano*1, 2,8

${ }^{1}$ Graduate School of Pharmaceutical Sciences, ${ }^{2}$ Graduate School of Medicine, and ${ }^{3}$ Drug Discovery Initiative, The University of Tokyo, Tokyo 113-0033, Japan

${ }^{4}$ Institute for Integrated Cell-Material Sciences (WPI-iCeMS), Kyoto University, Kyoto, 606-8501, Japan

5JST PRESTO, Tokyo, 102-0075, Japan

${ }^{6}$ Faculty of Science, Yamaguchi University, Yamaguchi, 753-8512, Japan.

7International Research Center for Neurointelligence, The University of Tokyo, 1130033 Tokyo, Japan

${ }^{8}$ CREST (Japan) Agency for Medical Research and Development (AMED), Tokyo 1000004, Japan

Corresponding authors e-mails:

Tasuku Ueno: tasueno@mol.f.u-tokyo.ac.jp

Yasuteru Urano: uranokun@m.u-tokyo.ac.jp

\begin{abstract}
Actin is a ubiquitous cytoskeletal protein, forming a dynamic network that generates mechanical forces in the cell. Here, in order to dissect the complex mechanisms of actin-related cellular functions, we introduce two powerful tools based on a new class of actin-binding small molecule: one enables visualization of the actin cytoskeleton, including super-resolution imaging, and the other enables highly specific green-light-controlled fragmentation of actin filaments, affording unprecedented control of the actin cytoskeleton and its force network in living cells.
\end{abstract}




\section{Manuscript}

Actin filaments are major components of the cytoskeleton in eukaryotic cells, functioning to maintain the shape and internal framework of cells, and to provide the cells with a driving force for shape change and movement ${ }^{1}$. Natural actin-binding small-molecular inhibitors have long been recognized as valuable tools for dissecting the mechanisms of actin-related cellular functions ${ }^{2}$. Actin-binding small molecules have also been utilized as platforms for functional molecules; for example, fluorescent phalloidin conjugate was originally developed in $1979^{3}$, and is still the gold standard for labeling endogenous actin filaments in fixed samples. Nevertheless, there is still a need to develop improved fluorescent actin-binding molecular conjugates for actin visualization, especially for application to real-time imaging in living cells and organisms 4,5 .

Here, we report that HMRef, a simple rhodol derivative bearing a hydroxymethyl group, provides a new, powerful tool for actin labeling in live cells (Fig. 1a, 1b). HMRef was originally developed as a fluorophore for in vivo tumor imaging probes ${ }^{6}$. Unexpectedly, we found that HMRef, which is highly fluorescent in aqueous media and is membrane-permeable, can clearly visualize the actin cytoskeleton, despite having no structural similarity to any of the known actin-binding natural products, such as phalloidin or jasplakinolide ${ }^{2}$. In order to elucidate the mechanism underlying the visualization of cellular actin fibers by HMRef, we first examined the in vitro interaction of HMRef with isolated F-actin/G-actin by means of fluorescence polarization (FP) assay. As expected, the FP signal was increased when HMRef was incubated with isolated F-actin, i.e., HMRef has intrinsic binding affinity for F-actin, and does not require assistance from actin-binding proteins (Fig. 1c). The FP signal increase was negligible in the presence of monomeric actin, suggesting specific binding of HMRef to polymeric actin. Secondly, we conducted competitive binding assay with HMRef and natural F-actin stabilizers using fixed HeLa cells (Supplementary Fig. 1). Both phalloidin and jasplakinolide significantly and dosedependently decreased the HMRef fluorescence response to actin in fixed cells, suggesting that their binding sites are likely to overlap, at least partially, with the HMRef binding site. HMRef may bind relatively weakly to F-actin as the natural products could compete with HMRef at lower concentrations. We also found that HMRef has little effect on actin polymerization or stability under our experimental conditions (Supplementary Fig. 2), which is consistent with a relatively weak interaction. Finally, we designed and synthesized a series of HMRef derivatives and applied them to live and fixed cells (Supplementary Fig. 3,4, Supplementary table 
1, and Supplementary Notes 1,2 ). Most of the HMRef derivatives also bound to Factin in fixed cells, but to a lesser extent. Of the 9 derivatives tested, HMRef seems to be the most suitable fluorescent probe for imaging the actin cytoskeleton in living cells.

We next confirmed that HMRef is suitable for super-resolution imaging techniques, STED $^{7,8}$ or SRRF, ${ }^{9}$ and indeed, we obtained highly detailed actin staining images (Fig. 1d, 1e and supplementary Fig. 5). Actin is one of the most highly conserved proteins throughout evolution, and so it was not unexpected that HMRef stains actin in multiple cell lines derived from various species (Fig. 1f and supplementary Fig. 6a-c), including Vero and Cos7 cells, for which SiR-actin does not work well ${ }^{5}$. Cell viability was not altered under the imaging conditions, but we found that the cell shape was highly disrupted at a tenfold higher concentration of HMRef (Supplementary Fig. 7). We also applied HMRef for imaging primary-cultured cells and tissues. HMRef clearly visualized retrograde actin flow in migrating fish keratocytes (Supplementary Fig. 8, and Supplementary Movie 1) ${ }^{10}$, as well as the contractile ring dynamics of cleavage furrow ingression during cell division in Drosophila wing disc (Fig. 1g, 1h). These results indicate that HMRef is a powerful tool to visualize the actin cytoskeleton of various cells in living cells and organisms in real time, without affecting the cell behaviors.

In the following, we utilized HMRef as a F-actin-binding scaffold to develop a functional probe for F-actin manipulation. A technique that can ablate the actin filament network with single-cell resolution would have great potential to better define the forces involved in adhesion and migration of multicellular assemblies. Although actin-targeting natural product inhibitors are useful tools in actin-related cell biology ${ }^{2}$, their spatial resolution is limited. One approach to circumvent this issue is the use of chromophore-assisted light inactivation (CALI), in which a suitable ligand serves to direct a chromophore specifically to the protein of interest, followed by light-induced release of reactive oxygen species to trigger spatiotemporally controlled inactivation of target molecules in situ 1112 .

Aiming to apply this strategy to actin, we have developed a new small-molecular

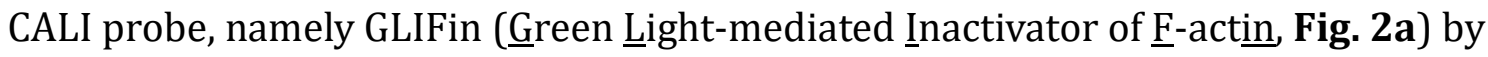
iodinating the xanthene core of HMRef in order to increase its singlet oxygen generation by making use of the so-called internal heavy-atom effect.13, 14 We confirmed the singlet oxygen-generating ability (Fig. 2b) and the F-actin-binding ability (Supplementary Fig. 9) of GLIFin, and then tested the ability of GLIFin to induce degradation of the actin cytoskeleton in cells upon laser irradiation (Fig. 2c, 
2d). As expected, GLIFin mediated photoinactivation of the actin filaments of cells in a light-power- and GLIFin-concentration-dependent manner (Supplementary Fig. 10), while causing no apparent damage to microtubules, which lie adjacent to actin filaments ${ }^{15}$, at least under our experimental condition (Supplementary Fig. 11). Factin fragmentation was not induced by either HMRef, actin-binding fluorophore (i.e., a much less efficient photosensitizer), or EosinY, a non F-actin-directing photosensitizer (Fig 2d). It is noteworthy that actin fragmentation proceeded in the dark on a time scale of an hour after irradiation (Supplementary Fig. 12 and Supplementary note 3). As the path length of singlet oxygen is as short as $<100 \mathrm{~nm}$ in cells ${ }^{16}$, GLIFin could achieve single-cell-level spatial resolution for actin fragmentation (Fig. 2e). We found that F-actin filaments recovered after a day (Fig. 2f), and cell viability was unaffected by GLIFin-mediated photoinactivation in the concentration range of $300 \mathrm{nM}$ or less (Supplementary Fig. 13).

We next applied GLIFin to epithelial cell monolayers (Fig. 2g and Supplementary Fig. 14) where cell-cell interaction involving the actin cytoskeleton at adherens junctions and actin-extracellular matrix (ECM) interplay mediate coordinative cell movement and morphogenesis ${ }^{17}$. Migration measurements showed a severe decrease in migration speed, in a light- and GLIFin-dependent manner (Fig. 2h), without loss of cell viability (Supplementary Fig. 15). Notably, the effect of GLIFinmediated inactivation was relatively long-lasting, and the migration rate was restored only after $\sim 12 \mathrm{hr}$ (Supplementary Notes 4). Interestingly, a limited forward movement of cells into open space was frequently observed in nonirradiated areas, where the cells formed a kind of "boundary layer", even though the space in front of actin-inactivated cells was still available (Fig. 2i). These observations might reflect disorder of long-range interactions via the intracellular actin network. Finally, we confirmed the applicability of GLIFin-mediated inactivation to an in vivo model, the wing disk of Drosophila larvae (Supplementary Fig. 16). Compared to the selective removal of cells by laser ablation, GLIFinmediated photoinactivation offers the advantage of low invasiveness. Specifically, GLIFin allows flexible disruption of intracellular force transmission while leaving cells alive and adhesive. We believe it will be a powerful tool in a variety of fields, including the study of supracellular organization to generate force between leader and follower cells during the cooperative movement of groups of cells ${ }^{18}$.

In present study, we have developed a new class of live cell visualization and manipulation probes for F-actin that are membrane-permeable and highly selective for F-actin at the single-cell level of resolution. We believe they complement existing 
visualization/manipulation techniques in our endeavor toward comprehensive understanding of actin function.

\section{References}

1. Pollard, T.D. \& Cooper, J.A. Science 326, 1208-1212 (2009).

2. Peterson, J.R. \& Mitchison, T.J. Cell Chem. Biol. 9, 1275-1285 (2002).

3. Wulf, E., Deboben, A., Bautz, F.A., Faulstich, H. \& Wieland, T. Proc. Natl. Acad. Sci. USA 76, 4498-4502 (1979).

4. $\quad$ Riedl, J. et al. Nat. Methods 5, 605-607 (2008).

5. Lukinavicius, G. et al. Nat. Methods 11, 731-733 (2014).

6. Asanuma, D. et al. Nat. Commun. 6, 6463 (2015).

7. Klar, T.A., Jakobs, S., Dyba, M., Egner, A. \& Hell, S.W. Proc. Natl. Acad. Sci. USA 97, 8206-8210 (2000).

8. Hell, S.W. \& Wichmann, J. Opt. Lett. 19, 780-782 (1994).

9. Gustafsson, N. et al. Nat. Commun. 7, 12471 (2016).

10. Lee, J., Ishihara, A., Theriot, J.A. \& Jacobson, K. Nature 362, 167-171 (1993).

11. Jay, D.G. Proc. Natl. Acad. Sci. USA 85, 5454-5458 (1988).

12. Jacobson, K., Rajfur, Z., Vitriol, E. \& Hahn, K. Trends Cell Biol. 18, 443-450 (2008).

13. McClure, D.S. J. Chem. Phys. 17, 905-913 (1949).

14. Gorman, A. et al. J. Am. Chem. Soc. 126, 10619-10631 (2004).

15. C.Rodriguez, O. et al. Nat. Cell Biol. 5, 599-609 (2003).

16. Moan, J. J. Photochem. Photobiol. B, Biol. 6. 343-344 (1990).

17. Ladoux, B. \& Mege, R.M. Nat. Rev. Mol. Cell Biol. 18, 743-757 (2017).

18. Vitorino, P. \& Meyer, T. Genes Dev. 22, 3268-3281 (2008). 
Figure 1. HMRef as a fluorescent probe for F-actin
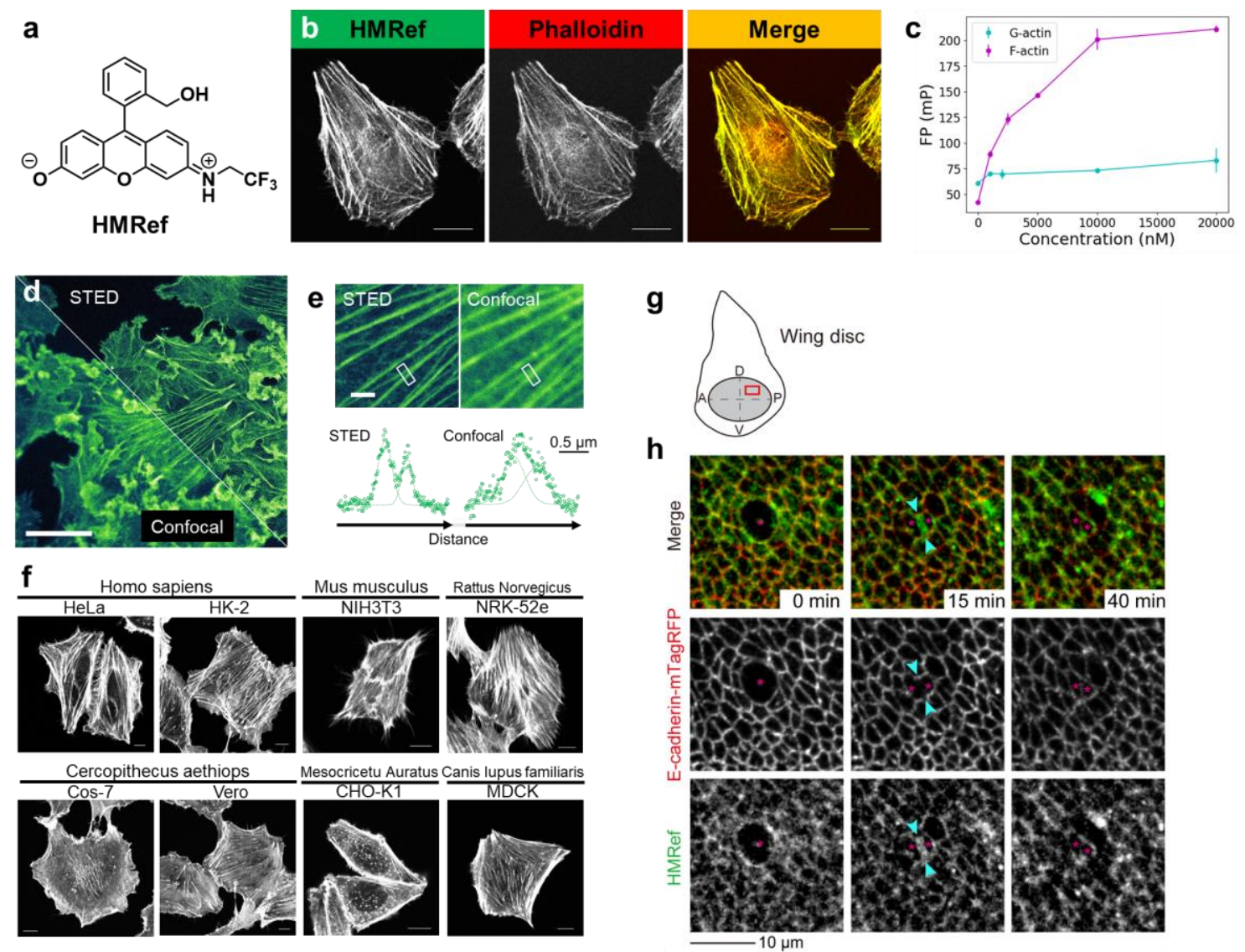

$-10 \mu \mathrm{m}$

(a) Structure of HMRef. (b) Confocal imaging of fixed and permeabilized HeLa cells stained with HMRef (green) and Alexa Fluor ${ }^{\mathrm{TM}} 647$ phalloidin (red). Fixed cells were incubated with 2U/mL Alexa Fluor ${ }^{\mathrm{TM}} 647$ phalloidin in DPBS for $30 \mathrm{~min}$, and then stained with $500 \mathrm{nM}$ HMRef in DPBS for $2 \mathrm{hr}$. Scale bar, $20 \mu \mathrm{m}$. (c) Evaluation of F/G-actin-binding ability of HMRef by means of fluorescence polarization. Values are mean \pm S.D., $n=2$ (F-actin, $1 \mathrm{nM}$ ) or 3 (others). (d) Stimulated emission depletion ("STED") imaging of living COS-7 cells stained with $1 \mu \mathrm{M}$ HMRef. A confocal image without STED ("confocal") is shown for comparison. Scale bar, $20 \mu \mathrm{m}$. (e) Magnified images and line profiles of HMRef-stained COS-7 cells. Line profiles show the fluorescence signal along the long axes in the insets, and the fitted Gaussian curves are also shown. Scale bar, $2 \mu \mathrm{m}$. (f) Confocal images of HMRef-loaded cells. $500 \mathrm{nM}$ HMRef, 30 min. Scale bar, $10 \mu \mathrm{m}$. (g) Schematic illustration of Drosophila wing disc. In (h), the posterior-dorsal region of the wing disc is imaged (red square). (h) Live imaging of Drosophila wing imaginal discs incubated with 500 nM HMRef. Selected 
snapshots from a movie showing HMRef (green in top panels, gray in bottom panels) and E-cad-mTagRFP (red in top panels, gray in middle panels). HMRef labels F-actin along the cell-cell junction and the contractile ring during cytokinesis (cyan arrowheads). Magenta asterisks indicate a dividing cell and its sister cells. Scale bar, $10 \mu \mathrm{m}$.

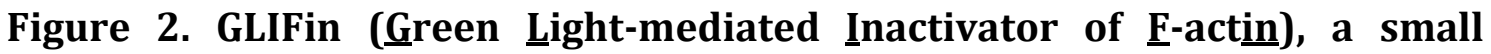
molecular CALI probe for F-actin
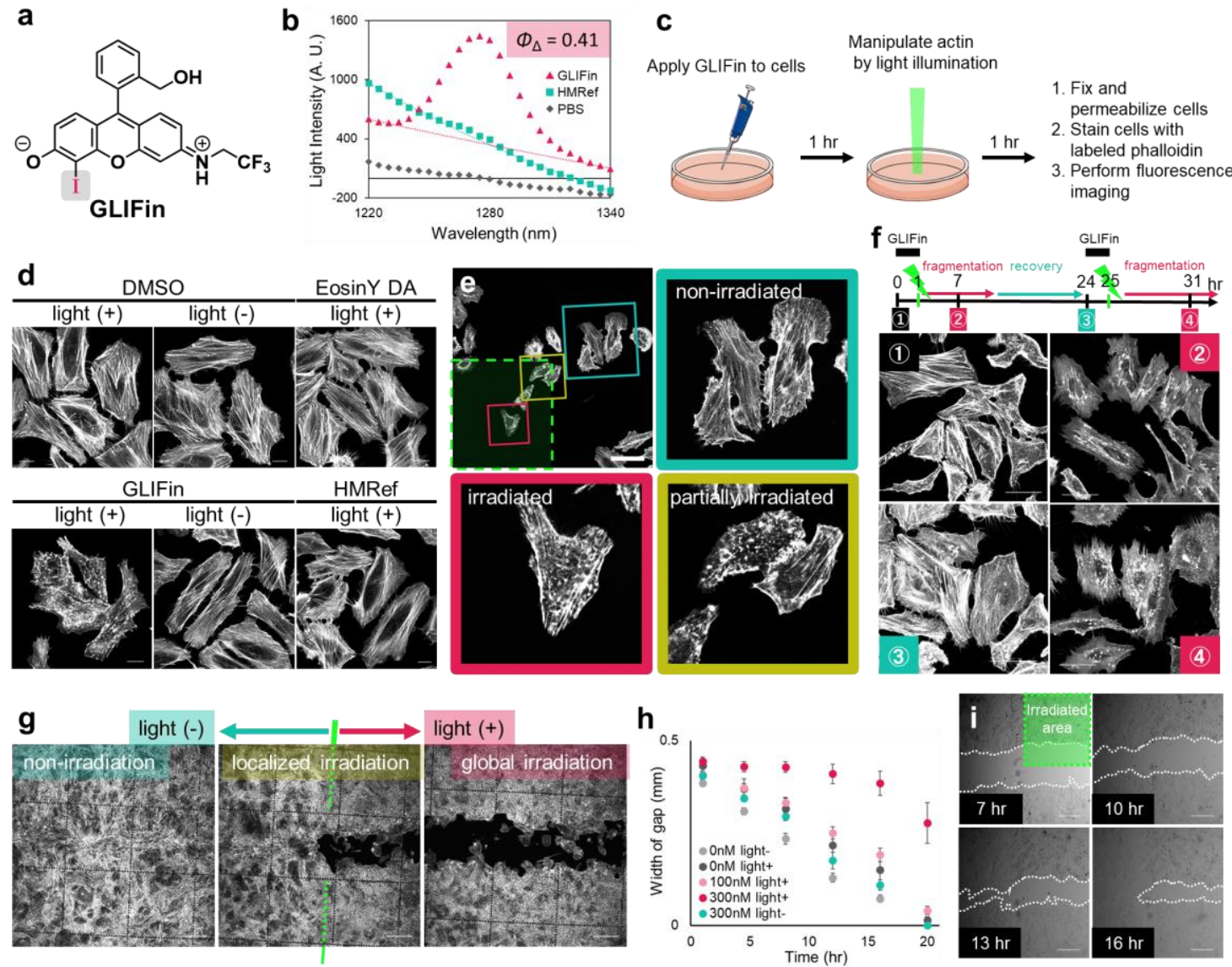

(a) Structure of GLIFin. (b) Representative luminescence spectra of ${ }^{1} \mathrm{O}_{2}$ generated in response to $508 \mathrm{~nm}$ laser illumination. $\Phi_{\Delta}$ - the quantum yield of ${ }^{1} \mathrm{O}_{2}$ generation. Dye concentration, $5 \mu \mathrm{M}$ in PBS. (c) Schematic illustration of the protocol for F-actin manipulation upon laser irradiation. (d) Effect of GLiFin-mediated light inactivation of F-actin. Cells were incubated with growth medium containing $300 \mathrm{nM}$ probes/vehicle for $1 \mathrm{hr}$, followed by illumination with green light $\left(27.0 \mathrm{~mW} / \mathrm{cm}^{2}\right.$ at BP515-569 nm, $1 \mathrm{~min}$ ), and F-actin was visualized with Alexa Fluor ${ }^{\mathrm{TM}} 647$. Scale bar: 
$10 \mu \mathrm{m}(\mathrm{e}) \mathrm{F}$-actin inactivation with single-cell resolution. Inactivation of a predefined localized area (green dashed line) was done by illumination with an argon laser (24.1 mW/ $\mathrm{cm}^{2}$ at $\left.514 \mathrm{~nm}, 1 \mathrm{~min}\right)$. After $1 \mathrm{hr}$, images were acquired. The green, red and yellow windows show enlarged views of the indicated region. Scale bar, 100 $\mu \mathrm{m}$. (f) Time-dependent recovery of F-actin inactivated with GLIFin. Cells were incubated with growth medium containing $300 \mathrm{nM}$ GLIFin for $1 \mathrm{hr}$, followed by illumination with green light $\left(22.6,23.5 \mathrm{~mW} / \mathrm{cm}^{2}\right.$ at $\left.514 \mathrm{~nm}, 1 \mathrm{~min}\right)$, and incubated, fixed and repeatedly irradiated at the indicated time points. F-actin was visualized with Alexa Fluor ${ }^{\mathrm{TM}}$ 647. Scale bar, $20 \mu \mathrm{m}$. (g) Actin cytoskeleton of cells from locally irradiated MDCK monolayer migration assay. Locally irradiated $\left(24.1 \mathrm{~mW} / \mathrm{cm}^{2}\right)$ MDCK cells were incubated for $12 \mathrm{hr}$, fixed and permeabilized, and then stained with Alexa FluorTM 647 phalloidin. Scale bar, $200 \mu \mathrm{m}$. (h) Time-dependent gap closure. Values are mean \pm S.D., $n=2$ (GLIFin $300 \mathrm{nM}$, light (-)) or 3 (others). (i) Asymmetric locomotion in a cell monolayer triggered by local inactivation. An MDCK monolayer was prepared as described above, followed by local light irradiation $\left(24.1 \mathrm{~mW} / \mathrm{cm}^{2}\right.$, indicated area) for $1 \mathrm{~min}$. Scale bar, $200 \mu \mathrm{m}$.

\section{Supplementary information}

Supplementary Figure1-18, Supplementary Movie1, Supplementary Table 1, and Supplementary Note 1-4.

\section{ORCID of corresponding authors}

Tasuku Ueno: 0000-0001-6657-8209

Yasuteru Urano: 0000-0002-1220-6327

\section{Conflict of Interest}

The authors declare no competing financial interests.

\section{Acknowledgement}

This research was supported in part by AMED under grant number JP19gm0710008 (to Y.U.), by Japan Science and Technology Agency (PRESTO, JPMJPR17P1 to D.A.), by MEXT/JSPS KAKENHI grants JP16H02606 and JP19H05632 (to Y.U.), 16H06574, 26750369, 17K14511 (to T.U.), 17H04764(to D.A.), $19 H 04935$ (to Y.I.), and by JSPS Core-to-Core Program, A. Advanced Research Networks (JPJSCCA20170007 to Y.U.). 


\section{Methods}

\section{Cell lines and culture.}

All cell lines were grown in DMEM, RPMI 1640, or F-12 containing $10 \%$ fetal bovine serum (FBS), $100 \mu \mathrm{g} / \mathrm{ml}$ penicillin and $100 \mu \mathrm{g} / \mathrm{ml}$ streptomycin (all reagents were purchased from Life Technologies). All cell lines were maintained at $37{ }^{\circ} \mathrm{C}$ in $5 \% \mathrm{CO}_{2}$. Details of the cell sources and culture media are given in Supplementary note 4.

\section{Imaging of the actin cytoskeleton stained with HMRef derivatives and/or fluorescence-labeled phalloidin in fixed cells.}

Cells were plated on glass-bottomed eight-chamber plates (Ibidi, 80826) and incubated with growth medium for one day. The cells were washed with PBS 3 times, then fixed and permeabilized with PBS containing 4\% HCHO and 0.1\% Triton-X for 10 min. PBS was removed, and the fixed cells were washed with PBS 3 times and incubated in PBS containing 0.66\% MeOH and $2 \mathrm{U}$ mL Alexa Fluor ${ }^{\mathrm{TM}} 647$ phalloidin (Thermo Fisher Scientific, A22287) for $30 \mathrm{~min}$. For co-staining with HMRef derivatives, the Alexa Fluor ${ }^{\mathrm{TM}} 647$ solution was replaced with PBS containing 500 $\mathrm{nM}$ or $1 \mu \mathrm{M}$ HMRef derivatives. Unless otherwise mentioned, fluorescence images were acquired with a confocal fluorescence microscope (TCS SP8, Leica) equipped with a multi-wavelength argon and He-Ne laser, and an objective lens (HCX PL APO CS 40x/1.25 Oil, Leica). The excitation and emission wavelengths were $488 \mathrm{~nm} / 510$ $550 \mathrm{~nm}$ for HMRef derivatives, and $633 \mathrm{~nm} / 661-750 \mathrm{~nm}$ for Alexa Fluor ${ }^{\mathrm{TM}} 647$ phalloidin.

\section{Fluorescence Polarization analysis. ${ }^{19}$}

Actin (1 mg) from rabbit muscle (Sigma-Aldrich, A2522-1MG) was dissolved in $1 \mathrm{~mL}$ of general actin buffer (Cytoskeleton Inc. Cat. \# BSA01-010; 'G-buffer'). The actin solution was left on ice for $1 \mathrm{hr}$ for depolymerization. Then $100 \mu \mathrm{L}$ of actin polymerization buffer (Cytoskeleton Inc. Cat. \# BSA02-001; 'P-buffer') and $200 \mathrm{nmol}$ ATP in $2.0 \mu \mathrm{L} \mathrm{H}_{2} \mathrm{O}$ were added and mixed. After $2 \mathrm{hr}$ of incubation for polymerization, a dilution series of the F-actin solution was prepared. To each solution, HMRef (final conc., $100 \mathrm{nM}$ ) was added and aliquots of the mixtures were pipetted into sample tubes. The fluorescence polarization was measured using a BEACON ${ }^{\mathrm{TM}} 2000$ (Pan Vera). 


\section{Pyrene-labeled actin polymerization assay.}

Polymerization assay was carried out as previously described ${ }^{5}$. G-buffer $(5 \mathrm{mM}$ Tris-HCl (pH 8.0), 0.2 mM CaCl 2 and 0.2 mM ATP) and P-buffer (100 mM Tris HCl, 20 $\mathrm{mM} \mathrm{MgCl}$, $500 \mathrm{mM} \mathrm{KCl}, 10 \mathrm{mM} \mathrm{ATP}, 50 \mathrm{mM}$ guanidine carbonate $\mathrm{pH}$ 7.5) were prepared according to the manufacturer's protocol. Briefly, a stock solution of 20 $\mathrm{mg} / \mathrm{mL}(465 \mu \mathrm{M})$ pyrene-labeled G-actin (Cytoskeleton Inc., cat. \# AP05-A) was 46.5-fold diluted with G-buffer, then centrifuged for $15 \mathrm{~min}$ at $15000 \mathrm{rpm}, 4^{\circ} \mathrm{C}$, and the supernatant was collected, providing $10 \mu \mathrm{M}$ working solution. The working solution was left on ice for $1 \mathrm{hr}$ for depolymerization and pipetted into wells of a black 384-well assay plate (10 $\mu \mathrm{L} /$ well) (Greiner Bio-one, cat 784900). $1 \mu \mathrm{L}$ of probe solution in DMSO was added mixed, and incubated for $10 \mathrm{~min}$, then $1 \mu \mathrm{L}$ of P-buffer and $20 \mathrm{nmol}$ ATP in $0.2 \mu \mathrm{L} \mathrm{H}_{2} \mathrm{O}$ were added and mixed. The time course of the fluorescence $(E x / E m=365 \mathrm{~nm} / 407 \mathrm{~nm})$ was measured using a Multilabel plate reader (EnVision 2103, PerkinElmer).

\section{Pyrene-labeled actin depolymerization assay.}

Depolymerization assay was carried out as previously described ${ }^{5}$. Briefly, a $10 \mu \mathrm{M}$ working solution of pyrene-labeled G-actin was prepared and pipetted into wells of a black 96-well assay plate ( $40 \mu \mathrm{L} /$ well). $10 \mu \mathrm{L}$ of P-buffer and $20 \mathrm{nmol}$ ATP in 0.2 $\mu \mathrm{L} \mathrm{H}_{2} \mathrm{O}$ were added and mixed. The actin solution was incubated for $2 \mathrm{hr}$ for polymerization. Then, $1 \mu \mathrm{L}$ of probe solution in DMSO was added and mixed. After incubation for $5 \mathrm{~min}$, the actin solution was 5 -fold diluted with $160 \mu \mathrm{L}$ G-buffer. The time course of the fluorescence $(\mathrm{Ex} / \mathrm{Em}=365 \mathrm{~nm} / 407 \mathrm{~nm})$ was measured using a Multilabel plate reader (EnVision 2103, PerkinElmer).

\section{STED microscopy. 7,8}

COS-7 cells were cultured on glass-bottomed dishes (Matsunami Glass D11531H) at $37{ }^{\circ} \mathrm{C}$ in $5 \% \mathrm{CO}_{2}$ in DMEM (Wako 045-30285) with 10\% fetal bovine serum (SIGMA 172012), 2\% L-glutamine solution (Wako 073-05391), 1\% sodium pyruvate solution (Wako 190-14881), and 1\% penicillin-streptomycin mixed solution (Nacalai 26253-84). The cells were gently washed twice with HEPES-buffered saline, pH 7.4 (HBS) (25 mM HEPES, $115 \mathrm{mM} \mathrm{NaCl}, 2.5 \mathrm{mM} \mathrm{KCl}, 2.0 \mathrm{mM} \mathrm{CaCl}$, $1.0 \mathrm{mM} \mathrm{MgCl} 2$, $25 \mathrm{mM}$ glucose), and incubated at ambient temperature for $30 \mathrm{~min}$ in the dark in HBS containing $1 \mu \mathrm{M}$ HMRef. Imaging was performed on a TCS SP8 STED 3X microscope (Leica Microsystems) including a pulsed white-light laser for excitation, 
a 592-nm depletion laser for STED, and a HyD detector. HMRef-stained cells were observed with a 100x oil immersion objective (HC PL APO CS2 100x/1.40 OIL) in a field of view of $8,192 \times 8,192$ pixels with a pixel size of $10 \mathrm{~nm} \times 10 \mathrm{~nm}$. The excitation and emission wavelengths were $488 \mathrm{~nm}$ and 500-570 $\mathrm{nm}$, respectively.

\section{Live-cell imaging with HMRef derivatives.}

Cells were plated on eight-chamber plates (Ibidi, 80826) and incubated for a day before imaging, unless otherwise mentioned. Cells were incubated in growth medium containing indicated concentrations of HMRef derivatives for $30 \mathrm{~min}$, and differential interference contrast (DIC) and fluorescence images were acquired with a confocal fluorescence microscope (TCS SP8, Leica) equipped with an argon laser and an objective lens (HCX PL APO CS 40x/1.25 Oil, Leica). The excitation and emission wavelengths were $488 \mathrm{~nm} / 510-550 \mathrm{~nm}$ for HMRef derivatives and 594 $\mathrm{nm} / 615-750 \mathrm{~nm}$ for RFP. Scale bar, $100 \mu \mathrm{m}$.

\section{SRRF imaging. 9}

Dual-color SRRF imaging was performed on a spinning-disc confocal microscope. HMRef and AlexaFluor647-phalloidin excitation was conducted with a $488 \mathrm{~nm} / 150$ $\mathrm{mW}$ diode laser (LM-488-150 Andor) and a $637 \mathrm{~nm} / 140 \mathrm{~mW}$ diode laser (LM-637140, Andor), respectively. The two lasers were fiber-coupled (7 line laser combiner, multi-mode x2, single-mode x1, LC-ILE-700-M2-S1, Andor) to a spinning disk confocal unit (CR-DFLY505; Andor) equipped with a multi-band dichroic mirror (DFly laser Dichroic for 405/488/561/640). The fluorescence was processed with appropriate filter sets for HMRef (TR-DFLY-F525-050, Andor) and AlexaFluor647 (TR-DFLY-F700-075, Andor) to capture fluorescence images with a CCD camera (iXion Life 888, Andor), driven by Fusion software (ver 2.0, Andor). Images were taken using a $60 \times$ objective (APON60XOTIRF, NA:1.49, Olympus) mounted on an inverted microscope (IX83, Olympus) equipped with Z-drift compensator (IX3-ZDC2, Olympus).

\section{CCK8 assay}

HeLa cells were seeded in a plastic-bottomed 96-well plate (Greiner Bio-One, 655090 ) at a density of $7.6 \times 10^{4}$ cells per well. After $24 \mathrm{hr}$, the medium was aspirated and replaced with fresh medium containing various concentrations of probes (adjusted by diluting $10 \mathrm{mM}$ DMSO stock solution). After incubation for $\sim 20$ 
$\mathrm{hr}$, the medium was aspirated and replaced with medium containing 5\% Cell Counting Kit-8 (Dojindo, CK04). After further incubation for $1 \mathrm{hr}$, the absorbance at $405 \mathrm{~nm}$ was measured using a plate reader (EnVision 2103 Multilabel Reader, PerkinElmer), to determine the cell viability. Values from the wells containing cells without probe and without photoirradiation were taken as representing $100 \%$ living cells, and values from wells without cells were taken as representing $100 \%$ dead cells.

For GLiFin treatment, the cells were stained with GLiFin for $1 \mathrm{hr}$, followed by light irradiation through a rodscope from a Xe light source, MAX301 (BP515-569 nm) for $1 \mathrm{~min}$. The medium was replaced with $200 \mu \mathrm{L} /$ well of fresh medium. After $20 \mathrm{hr}$, CCK8 assay was performed as described above.

\section{HMRef imaging of Fish Keratocytes}

Keratocytes of Central American cichlids (Hypsophrys nicaraguensis) were cultured in culture medium (Leibovitz's medium: L-15, L5520: Sigma-Aldrich, St Louis, MO) supplemented with $10 \%$ fetal calf serum (Nichirei, Tokyo, Japan) and antibiotic/antimycotic solution (09366-44: Nacalai Tesque, Kyoto, Japan) as previously described ${ }^{20}$. All methods were carried out in accordance with national guidelines and the Regulation on Animal Experimentation at Yamaguchi University. All experimental protocols were approved by Yamaguchi University Animal Use Committee. Cells were treated with $0.5 \mathrm{~g} / \mathrm{L}$ trypsin and $0.53 \mathrm{mM}$ EDTA (trypsinEDTA, 32778-34: Nacalai Tesque) for 30-60 s to separate any cell-cell adhesions. The single keratocytes ware treated with the culture medium containing $250 \mathrm{nM}$ HMRef for $10 \mathrm{~min}$. Then, the medium was replaced with the culture medium containing no probe. The migrating keratocytes were observed using an inverted microscope (Ti; Nikon, Tokyo, Japan) equipped with a laser confocal scanner unit (CSU-X1; Yokogawa, Tokyo, Japan) with a 100× objective lens (CFI Apo TIRF 100×H/1.49; Nikon, Tokyo, Japan). The fluorescence images were detected using an EM CCD camera (DU897; Andor, Belfast, UK).

\section{HMRef time-lapse imaging of Drosophila wing disc}

Drosophila melanogaster larvae expressing E-cadherin-mTagRFP21 were dissected in Schneider's medium (Thermo Fisher 21720024) containing 5\% FBS (biowest, 
s1810). The wing discs were cultured in Schneider's medium in the presence of 500 nM HMRef on a $35 \mathrm{~mm}$ glass-based dish (IWAKI 3911-035). After incubation for 1 hr, time-lapse imaging was performed with an inverted confocal microscope (A1R; Nikon) equipped with a $60 \times /$ NA1.2 Plan Apochromat water-immersion objective. The excitation and emission wavelengths were $488 \mathrm{~nm} / 500-550 \mathrm{~nm}$ for HMRef, and $561 \mathrm{~nm} / 570-620 \mathrm{~nm}$ for E-cadherin-mTagRFP. Images were taken at $5 \mathrm{~min}$ interval for $65 \mathrm{~min}$ at $\sim 25^{\circ} \mathrm{C}$.

Image processing was performed by using Image J. Briefly, the HMRef and Ecadherin signals on the adherens junction plane were extracted by using a custommade macro. The background signal was subtracted using the "subtract background" command ( $r=50$ ) for the HMRef image.

\section{UV-vis Absorption and fluorescence spectroscopy.}

UV-visible absorption spectra were obtained on a Shimadzu UV-1800. Fluorescence spectra were acquired with a Hitachi F7000. The slit width was $1 \mathrm{~nm}$ for both excitation and emission. The photomultiplier voltage was $400 \mathrm{~V}$. Relative fluorescence quantum yields were obtained by comparing the area under the emission spectra of the test samples with standard samples and were calculated according to the following equation

$$
\Phi_{\mathrm{X}} / \Phi_{\mathrm{st}}=\left[A_{\mathrm{st}} / A_{\mathrm{x}}\right]\left[n_{\mathrm{X}}{ }^{2} / n_{\mathrm{st}}{ }^{2}\right]\left[D_{\mathrm{x}} / D_{\mathrm{st}}\right]
$$

where st = standard; $\mathrm{x}=$ sample; $\mathrm{A}=$ absorbance at the excitation wavelength; $\mathrm{n}=$ refractive index; and $\mathrm{D}=$ area under the fluorescence spectra on an energy scale. Optical properties of probes $(1 \mu \mathrm{M})$ were examined in $0.1 \mathrm{M}$ sodium phosphate buffer containing $0.1 \%$ DMSO as a cosolvent. For determination of fluorescence quantum efficiency $\left(\Phi_{\mathrm{fl}}\right)$, fluorescein in $0.1 \mathrm{M}$ aqueous $\mathrm{NaOH}\left(\Phi_{\mathrm{fl}}=0.85\right)$ was used as a standard 22 .

\section{Singlet oxygen detection by near-infrared spectroscopy}

Singlet oxygen was detected by measuring ${ }^{1} \mathrm{O}_{2}$ luminescence at around $1270 \mathrm{~nm}$ upon laser irradiation, using a near-infrared emission spectrometer (Fluorolog-3, Horiba, Japan.). Probe solution (PBS containing 0.1\% DMSO as a cosolvent) was excited with monochromatic light (508 $\mathrm{nm}$ ) and luminescence was recorded 
between $1220-1340 \mathrm{~nm}$ in $5 \mathrm{~nm}$ steps. To calculate the quantum yield of ${ }^{1} \mathrm{O}_{2}$ generation, the luminescence signal was integrated for 7 seconds for each wavelength. The quantum yield was calculated by using Rose bengal in PBS as a reference $(0.75)^{23}$.

\section{GLIFin-Mediated light inactivation of F-actin.}

Cells were prepared as described above. They were incubated in growth medium containing GLIFin for $1 \mathrm{hr}$, followed by light irradiation by using BP515-569 nm light from a Xe light source, MAX301 (Asahi Spectra Co., Ltd., for global irradiation) or a TCS SP8 (Leica, $514 \mathrm{~nm}$, for local irradiation). In experiments involving an incubation time of over $1 \mathrm{hr}$ after irradiation, the medium was replaced with fresh medium.

\section{Microtubule actin co-staining.}

After GLIFin-mediated light inactivation of F-actin as described above, cells were washed with PBS 3 times, then fixed and permeabilized with PBS containing 4\% HCHO and $0.1 \%$ Triton-X. After $10 \mathrm{~min}$, the solution was aspirated. The fixed cells were washed with PBS 3 times and blocked with 1\% bovine serum albumin (BSA)/PBS. After 30 min, the blocking solution was aspirated, and the fixed cells were incubated in PBS containing 1\% BSA, 0.66\% MeOH, 2 U/mL Alexa Fluor ${ }^{\mathrm{TM}} 647$ phalloidin (Thermo Fisher Scientific, A22287), $3 \mu \mathrm{g} \mathrm{mL}$ anti-alpha tubulin antibody conjugated with FITC (abcam, ab64503), and $3 \mu \mathrm{g} / \mathrm{mL}$ DAPI (Invitrogen, D1306) at ambient temperature for $1 \mathrm{hr}$. The PBS was aspirated and replaced with fresh PBS. Fluorescence imaging was done at $405 \mathrm{~nm} / 430-465 \mathrm{~nm}$ for DAPI, $488 \mathrm{~nm} / 510-550$ $\mathrm{nm}$ for FITC (tubulin), and $633 \mathrm{~nm} / 661-750 \mathrm{~nm}$ for Alexa 647 (F-actin).

\section{GLIFin manipulation of Drosophila wing cells}

The wing disc was dissected and mounted as described above and incubated with Schneider's medium containing $1 \mu \mathrm{M}$ GLIFin and 5\% FBS for $1 \mathrm{hr}$ prior to the light irradiation. To perform the light-mediated inactivation experiment, the wing disc was irradiated for $1.5 \mathrm{~min}$ with $488-\mathrm{nm}$ laser at 5\% power. Non-irradiated wing 
discs were used as a control. The control and irradiated wing discs were observed at $5 \mathrm{~min}$ before and at $3.5 \mathrm{hr}$ after the irradiation. To examine the effect of GLIFin manipulation on the F-actin intensity, the wing discs were fixed at room temperature for $30 \mathrm{~min}$ in PBS containing 4\% paraformaldehyde. After washing with PBS containing $0.1 \%$ Triton $\mathrm{X}-100$, these preparations were incubated overnight with Alexa Fluor ${ }^{\mathrm{TM}} 647$ phalloidin (1/1000, Thermo Fisher A22287). The E-cadherin and phalloidin signals on the adherens junction plane were extracted as described above.

\section{Epithelial cell sheet migration assay.}

MDCK cells ( $4.0 \times 10^{5}$ cells / $\mathrm{mL}$ ) were plated on both sides of eight-chamber plates (Ibidi, 80826 or 80206-G500) separated with 25 Culture-Inserts 2-well (Ibidi, 80209) and incubated in growth medium for one day. After removal of the medium from the cells, GLiFin-mediated light inactivation of F-actin was carried out. DIC images were captured with a confocal fluorescence microscope (TCS SP8, Leica). Fluorescence images were acquired as described above (also see Supplementary Fig 18).

\section{Live-dead staining.}

Live-dead staining was carried out according to the manufacturer's protocol. Briefly, cells were incubated in PBS containing 0.15\% DMSO, $2 \mu \mathrm{M}$ Calcein-AM (Thermo Fisher Scientific, L3224), $2 \mu \mathrm{M}$ ethidium homodimer-1 (Thermo Fisher Scientific, L3224), and $2 \mu \mathrm{g} / \mathrm{mL}$ DAPI (Invitrogen, D1306). Fluorescence images, (488 nm/ 510-570 nm for live, $561 \mathrm{~nm} / 650-750 \mathrm{~nm}$ for dead) were acquired with a confocal fluorescence microscope (TCS SP8, Leica) equipped with an argon laser and an objective lens (10x/0.40 dry, Leica). 


\section{References}

19. Lea, W.A. \& Simeonov, A. Expert Opin. Drug Discov. 6, 17-32 (2011).

20. Okimura, C., Taniguchi, A., Nonaka, S. \& Iwadate, Y. Sci. Rep. 8, 10615 (2018).

21. Pinheiro, D. et al. Nature 545, 103-107 (2017).

22. Paeker, C.A. \& Rees, W.T. Analyst 85, 587-600 (1960).

23. DeRosa, M.C. \& Crutchley, R.J. Coord. Chem. Rev. 233-234, 351-371 (2002). 